\title{
IMPACT OF KRAS VARIANT rS61764370 ON BREAST CANCER MORBIDITY
}

\author{
M. Ustinova, Z. Daneberga, D. Bērziņa, M. Nakazawa-Miklaševiča, J. Maksimenko, J. Gardovskis, \\ E. Miklaševičs* \\ Institute of Oncology, Riga Stradins University, Riga LV1007, Latvia
}

\begin{abstract}
Low-penetrance gene variants and their combinations are topical study objects in breast cancer pathogenesis. Single nucleotide polymorphism rs61764370, localized in 3' UTR of KRAS gene, plays an important role in the development and progression of several cancers. The aim of our study was to determine the $K R A S$ variant impact on breast cancer morbidity. Patients and Methods: 2214 patients diagnosed with breast cancer and 861 healthy controls were screened for $K R A S$ variant by RFLP method. Available clinical data were collected and processed using statistical analysis methods. Results of present study suggest the KRAS variant impact on breast cancer development risk in premenopausal women, but it has no effect on breast cancer prognosis. We did not observe any $K R A S$ variant effect on breast cancer patient 10-year disease-specific survival rates.
\end{abstract}

Key Words: breast cancer, rs61764370, KRAS variant, predisposing factor.

Single nucleotide polymorphism rs61764370 (GRCh38 38.1/141) localized in 3' UTR of the KRAS gene (MIM 190070) alters complementary site of KRAS inhibitory micro-ribonucleic acid let-7, thus hinders let-7-mediated regulation of the gene $[1,2]$. The results of in vitro studies describing the impact of the KRAS variant on cell processes are contradictory, setting two potential scenarios: overexpressed KRAS gene [1] or lower let-7 levels [3]. There are conflicting data about the KRAS variant effect on breast cancer biology [2, 4]. In a recent study from Ovarian Cancer Association Consortium and colleagues with performed rs61764370 genotyping for 140,012 women the authors reported no association of rs61764370 with risk of ovarian or breast cancer nor with clinical outcome [5]. Whereas McVeigh and colleagues described the KRAS variant as significant marker, which in combination with estrogen withdrawal increases breast cancer risk and predicts aggressive tumor biology [6].

\section{MATERIALS AND METHODS}

Study groups. The breast cancer patient group consisted of 2214 European descent women aged 24 to 95 years (mean age $60.35 \pm 12.59$ years), who have been diagnosed with breast cancer according to ICD-10. Patients with proven founder variants c. 181T>G (p.Cys61Gly), c.4035delA (p.Glu1346Lysfs) and c.5266dupC (p.GIn1756Profs) of the BRCA1 gene (MIM 113705) and/or positive family history of disease, indicating a possible presence of high penetrance variant, were excluded from the study group.

861 European descent voluntary women aged 18 to 92 years (mean age $47.63 \pm 19.07$ years) without any oncologic illnesses reported at the time of application were included in the control group. The distribution of cases and controls by age groups is shown in Table 1.

Submitted: August 10, 2015.

*Correspondence: E-mail: Edvins.Miklasevics@rsu.Iv; Fax: +37167069545
Table 1. Frequency of KRAS variant carriers among breast cancer patients and healthy women stratified by age

\begin{tabular}{ccccc}
\hline & \multicolumn{2}{c}{ Breast cancer patients } & \multicolumn{2}{c}{ Healthy women } \\
\cline { 2 - 5 } Age group & $\begin{array}{c}\text { Number } \\
\text { of patients, } \\
\mathrm{n}(\%)\end{array}$ & $\begin{array}{c}\text { Frequency } \\
\text { of KRAS mu- } \\
\text { tation, } \mathrm{n}(\%)\end{array}$ & $\begin{array}{c}\text { Number } \\
\text { of patients, } \\
\mathrm{n}(\%)\end{array}$ & $\begin{array}{c}\text { Frequency } \\
\text { of KRAS mu- } \\
\text { tation, } \mathrm{n}(\%)\end{array}$ \\
\hline$\leqslant 40$ years & $128(5.8)$ & $17(13.3)$ & $358(41.7)$ & $32(8.9)$ \\
$41-60$ years & $966(43.6)$ & $111(11.5)$ & $263(30.5)$ & $14(5.3)$ \\
$61-80$ years & $1015(45.9)$ & $122(12.0)$ & $194(22.5)$ & $22(11.3)$ \\
$>80$ years & $105(4.7)$ & $16(15.2)$ & $46(5.3)$ & $4(8.7)$ \\
\hline
\end{tabular}

Informed consent was obtained from all individual participants included in the study.

Genotyping of rs61764370. Genomic DNA was isolated from peripheral blood leukocytes using FlexiGene DNA Kit (Qiagen, Hilden, Germany). The presence of rs61764370 was detected by restriction fragment length polymorphism analysis. PCR was performed as described elsewhere [1]. Primer sequences used for PCR were as follows: forward $-5^{\prime}$-CCTGAGTAGCTGGGATTACA-3' reverse - 5'-GGATACCATATACCCAGTGCCTT-3'. Touch-down PCR was performed in following conditions: $96{ }^{\circ} \mathrm{C}$ for $10 \mathrm{~min}$ as initial denaturation, followed by 20 cycles of $96^{\circ} \mathrm{C}$ for $30 \mathrm{~s}, 63^{\circ} \mathrm{C}$ for $30 \mathrm{~s}$ and $72^{\circ} \mathrm{C}$ for $45 \mathrm{~s}$, reducing primer hybridization temperature $-0.5^{\circ} \mathrm{C}$ per cycle, prolonged with 20 cycles of $96{ }^{\circ} \mathrm{C}$ for $30 \mathrm{~s}, 53{ }^{\circ} \mathrm{C}$ for $30 \mathrm{~s}$ and $72{ }^{\circ} \mathrm{C}$ for $45 \mathrm{~s}$, with final elongation at $72^{\circ} \mathrm{C}$ for $10 \mathrm{~min}$. Amplification products were digested by restriction endonuclease Hinfl (Thermo Fisher Scientific, Waltham, Massachusetts, USA) and separated by $2 \%$ agarose gel electrophoresis.

Statistical analysis was performed with program $R$ version 3.1.0., statistical significance was set at 95\% level. The odds ratio was calculated by using "cc" function of Epicalc package. Chi square test was performed by using "chisq.test" function of Stat package for analysis of the distribution of KRAS variant in patients with various subtypes of breast cancer.

Ethical approval. All procedures performed in studies involving human participants were in accordance with the ethical standards of the institutional committee and with the 1964 Helsinki declaration and its later amendments or comparable ethical standards. 
Permission for the research project has been given by the Ethical Committee of Riga Stradins University.

\section{RESULTS}

The KRAS variant was detected in $12.01 \%$ (266/2214) breast cancer cases and 8.36\% (72/861) controls. Prevalence of rs61764370 variant among control subjects was similar to one in Europe $7.6 \%$ [1]. There was no statistically significant difference between the cancer stages $\left(X^{2}\right.$ test $=5.43, \mathrm{df}=3$, $p=0.14)$ and the mean age at the moment of diagnosis of the KRAS variant positive (60.72 years) and negative (60.31 years) breast cancer patients.

The higher likelihood of being the KRAS variant carrier for breast cancer patients compared to healthy women was found (case-control study, odds ratio $(\mathrm{OR})=1.50 ; 95 \%$ confidence interval $(\mathrm{Cl})=1.13-2.0$, $p=0.004$, statistical power $=85.09 \%)$. The same coherence was examined stratifying patients into different age groups. Although the frequency of KRAS variant in cases and controls divided by age groups is variable (Fig. 1), only women diagnosed with breast cancer at the age of 41-60 years demonstrate a higher probability of being carriers of the KRAS variant compared to controls at the same age $(\mathrm{OR}=2.30 ; 95 \%$ $\mathrm{Cl}=1.28-4.42 ; p=0.003)$. There were no similar associations found in other age groups (Table 2).

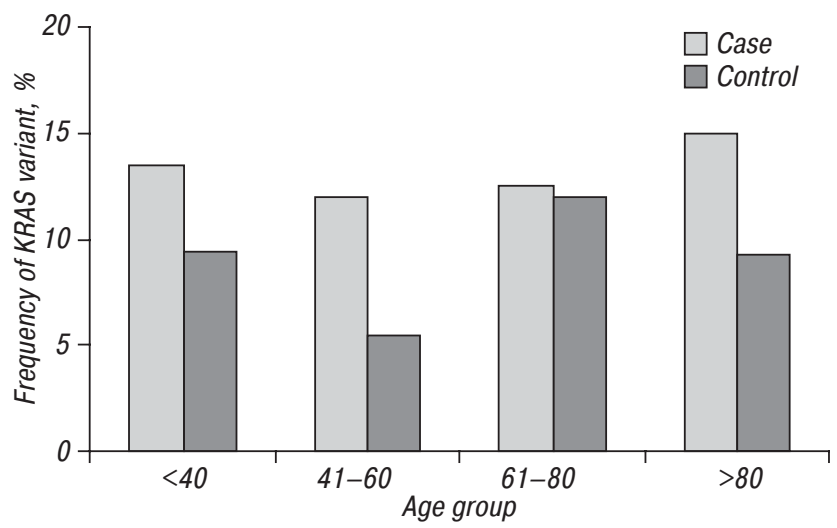

Fig. 1 Frequency of KRAS variant among cases and controls in different age groups

Table 2. Age stratified case-control study results

\begin{tabular}{cccc}
\hline Age group & OR & $95 \% \mathrm{Cl}$ & $\mathrm{p}$ value \\
\hline$\leqslant 40$ years & 1.55 & $0.78-3.01$ & 0.174 \\
$41-60$ years & 2.30 & $1.28-4.42$ & 0.003 \\
$61-80$ years & 1.06 & $0.65-1.80$ & 0.904 \\
$>80$ years & 1.88 & $0.56-8.21$ & 0.434 \\
\hline
\end{tabular}

The survival data were available for all breast cancer patients. Statistical analysis based on these data did not reveal any differences in disease-specific survival rates between $K R A S$ variant positive and negative breast cancer patients in 10 year observation period (log-rank test, $p=0.695$ ) (Fig. 2).

\section{DISCUSSION}

The results of our study suggest that the KRAS variant is slightly more frequent among breast cancer patients than it is in the healthy control group.

So far it has been reported that the KRAS variant significantly increases the risk of ovarian cancer, triple negative breast cancer and lung cancer $[1,4,7]$. Recent study of McVeigh and colleagues described the KRAS variant as significant marker, which in combination with estrogen withdrawal increases breast cancer risk and predicts aggressive tumor biology [6]. Our study demonstrated a slightly increased risk of developing breast cancer for KRAS variant positive women. After stratifying patients into different age groups it is noteworthy that the presence of the variant only in 41-60 years of age group was associated with increased morbidity. Although the data about hormonal levels in investigated patients were not obtained, it is tempting to speculate that the KRAS variant may be a predisposing factor for breast cancer development in association with hormonal fluctuations, which is a part of normal physiology for women in this age group. This finding of present study is in concert with results published by McVeigh and colleagues [6].

Ovarian Cancer Association Consortium and colleagues published the results based on large cohort study — no association of rs61764370 with risk of ovarian or breast cancer nor with clinical outcome [5]. Contradictions may be explained by the absence of stratification in the age groups.

KRAS variant association with premenopausal women in the context of triple negative breast cancer has been described before $[4,6]$. Our study shows an apparent association between KRAS variant and breast cancer risk among women at menopausal age, suggesting that this sequence variant may be indeed a predisposing factor for breast cancer development in association with hormonal fluctuations.

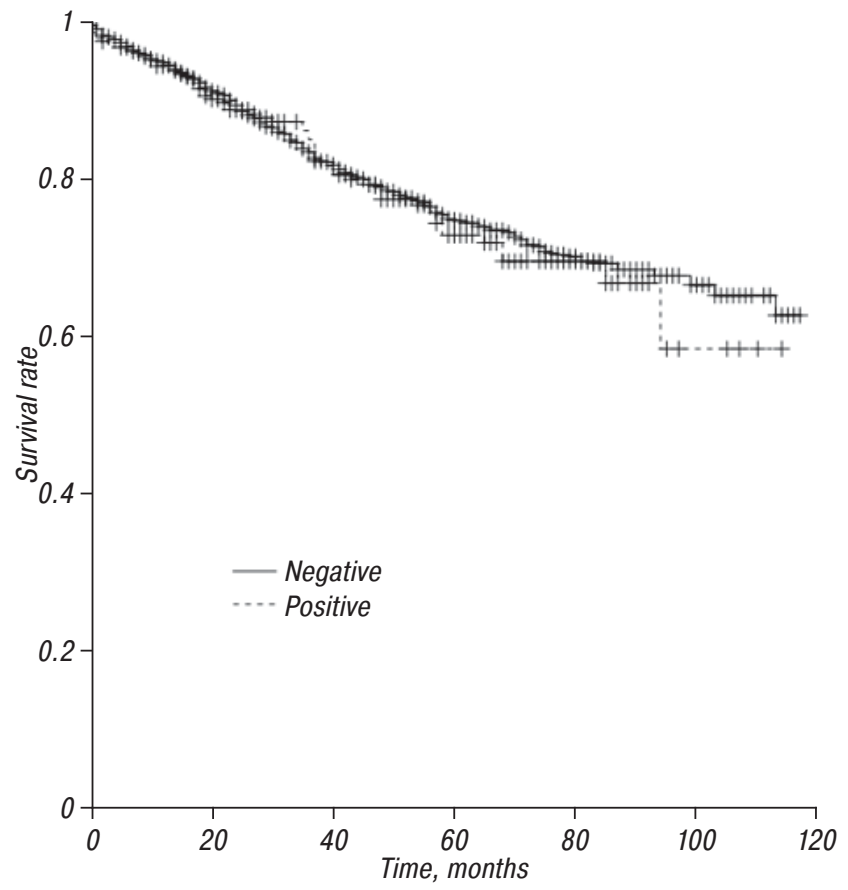

Fig. 2 10-year disease-specific survival rates for KRAS variant positive and negative breast cancer patients (Kaplan - Meier analysis)

The survival analysis showed no difference in disease-specific survival rate between the KRAS variant positive and negative breast cancer patients during 10 years long observation period (log-rank test, $p=0.695)$. This result indicates that the KRAS variant is involved in the breast cancer development in specific age group (41-60 years of age) but not in the prognosis. 
We carried analyses (data not shown) investigating proportion distribution of breast cancer molecular subtypes between KRAS variant positive and negative patients, as this variant allele has been previously associated with HER2-positive and triple negative tumors $[2,4,6]$. Our study results showed only a slight trend of increasing triple negative breast cancer cases among KRAS variant positive breast cancer patients and luminal $\mathrm{B}$ breast cancer cases among KRAS variant negative patients, though these data did not reach statistical significance.

Presented results imply the necessity for extended study of the KRAS variant frequency in combination with clinical data among premenopausal women.

\section{ACKNOWLEDGEMENTS}

This study was funded by European Social Fund grantNr. 2013/0047/1DP/1.1.1.2.0/13/APIA/VIAA/017.

\section{CONFLICT OF INTEREST STATEMENT}

The authors declare that they have no conflict of interests.

\section{REFERENCES}

1. Chin LJ, Ratner E, Leng S, et al. A SNP in a let-7 microRNA complementary site in the KRAS $3^{\prime}$ untranslated region increases non-small cell lung cancer risk. Cancer Res 2008; 68: 8535-40. doi: 10.1158/0008-5472.

2. Cerne JZ, Stegel V, Gersak K, et al. KRAS rs61764370 is associated with HER2-overexpressed and poorly-differentiated breast cancer in hormone replacement therapy users: a case control study. BMC Cancer 2012; 12: 105. doi: 10.1186/1471-2407-12-105.

3. Crowley EH, Arena S, Lamba S, et al. Targeted knockin of the Polymorphism rs61764370 does not affect KRAS expression but reduces let-7 levels. Hum Mutat 2014; 35: 208-14. doi: 10.1002/humu.22487.

4. Paranjape T, Heneghan H, Lindner R, et al. A 3'-untranslated region KRAS variant and triple-negative breast cancer: a case-control and genetic analysis. Lancet Oncol 2011; 12: 377-86. doi: 10.1016/S1470-2045(11)70044-4.

5. Hollestelle A, van der Baan FH, Berchuck A, et al. Ovarian cancer association consortium, breast cancer association consortium, consortium of modifiers of BRCA1 and BRCA2. No clinical utility of KRAS variant rs61764370 for ovarian or breast cancer. Gynecol Oncol 2015; pii: S00908258(15)00863-X. doi: 10.1016/j.ygyno.2015.04.034.

6. McVeigh TP, Jung SY, Kerin MJ, et al. Estrogen withdrawal, increased breast cancer risk and the KRAS-variant. Cell Cycle 2015; 14: 2091-9. doi: 10.1080/15384101.2015.1041694.

7. Ratner E, Lu L, Boeke M, et al. A KRAS-variant in ovarian cancer acts as a genetic marker of cancer risk. Cancer Res 2010; 70: 6509-15. doi: 10.1158/0008-5472.CAN-10-0689. 